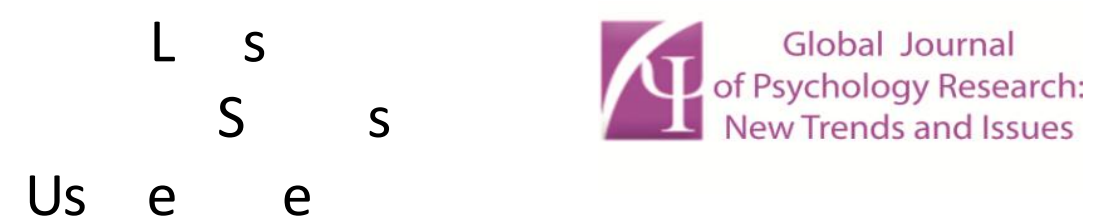

www.gipr.eu

\title{
Eating behaviour in relation to personality dimensions and self-esteem
}

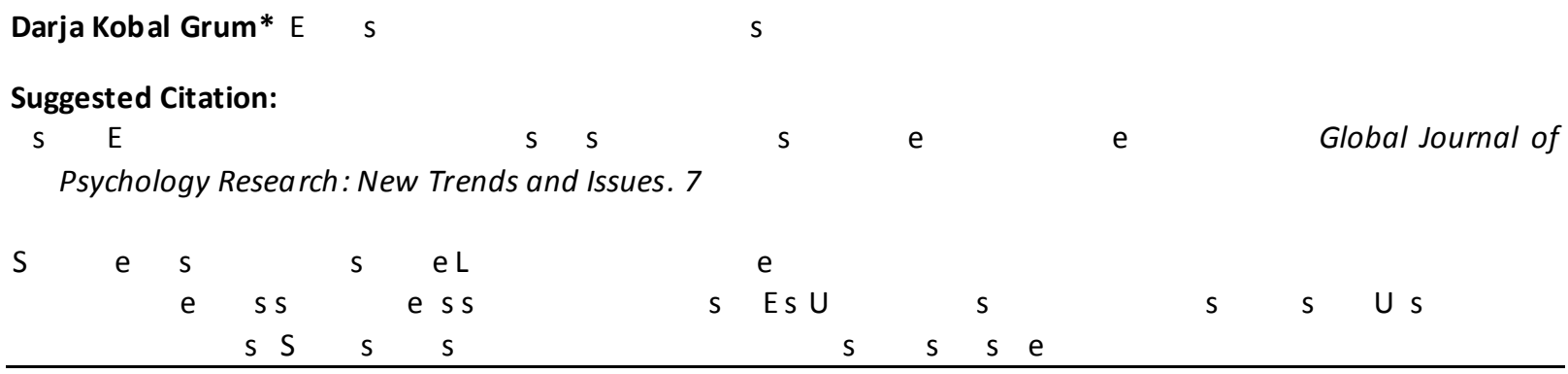

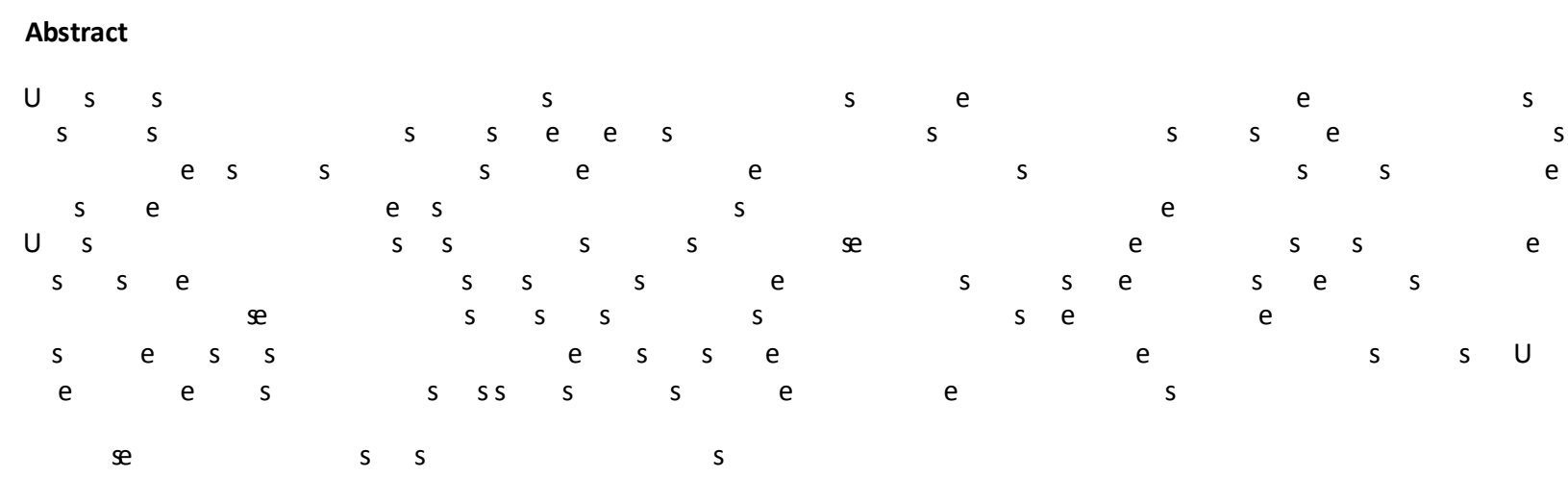

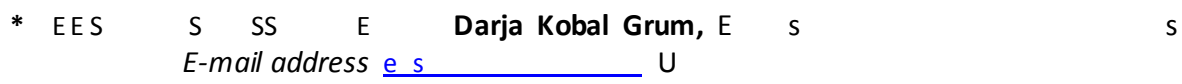


$\mathrm{E}$
Trends and Issues. 7

\section{Introduction}

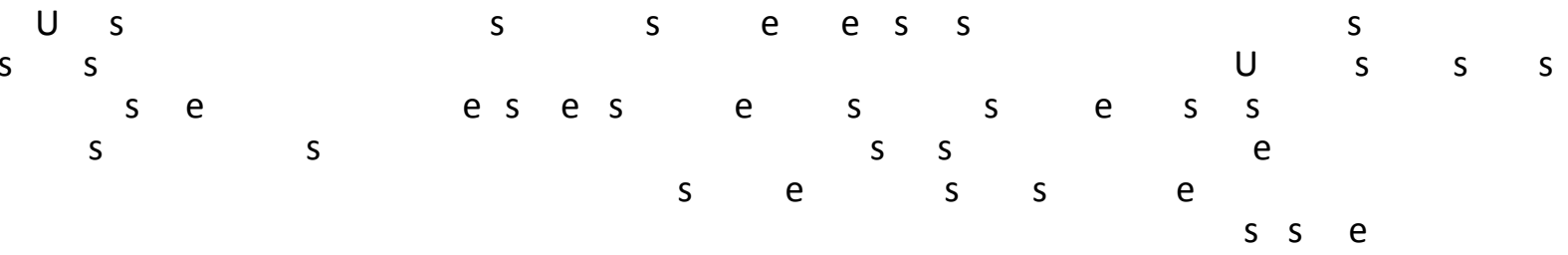

$$
\begin{aligned}
& \text { S S }
\end{aligned}
$$

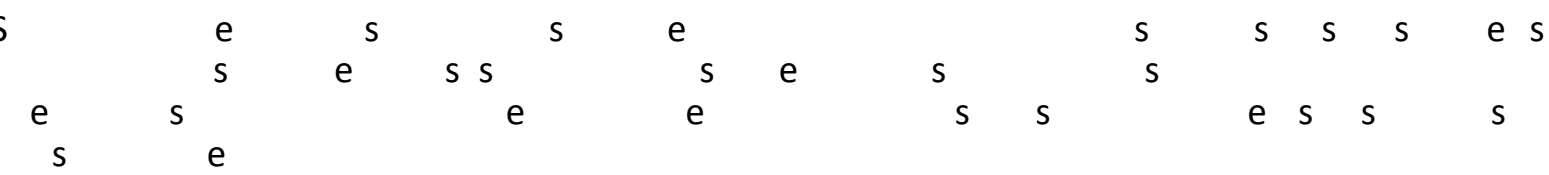

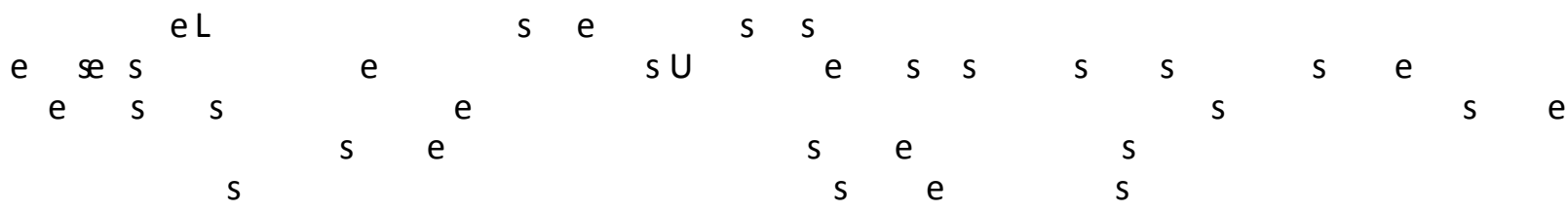

$$
\begin{aligned}
& \mathrm{S} S
\end{aligned}
$$

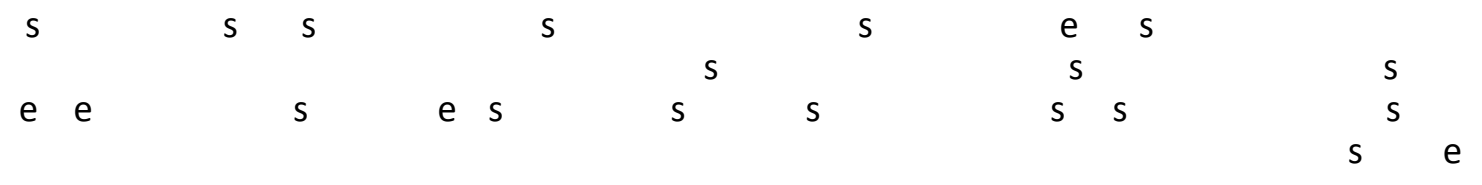

$$
\begin{aligned}
& \text { e } \quad \text { e } \quad s \quad s \\
& \begin{array}{lllllll}
e & s & s & e & e & s & \\
& & s & e & \text { se } s & s
\end{array}
\end{aligned}
$$

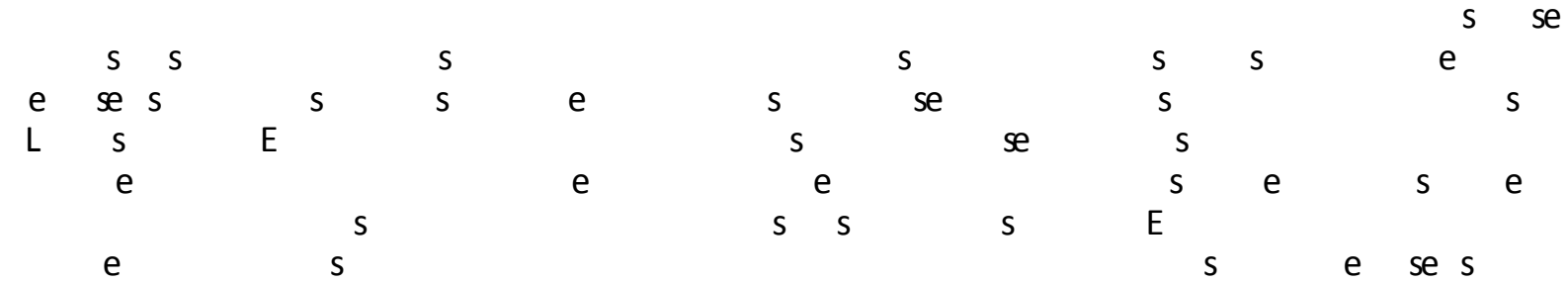

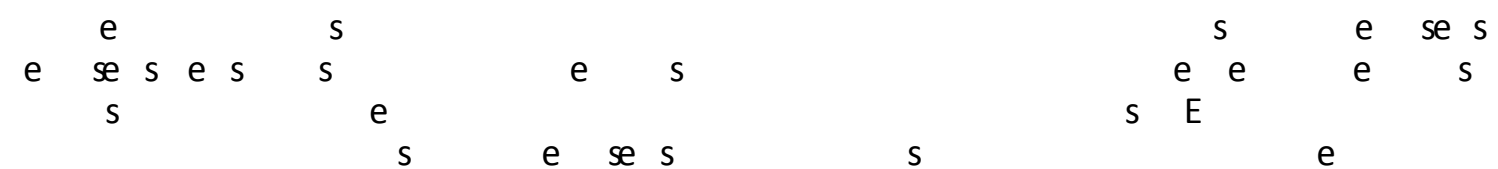

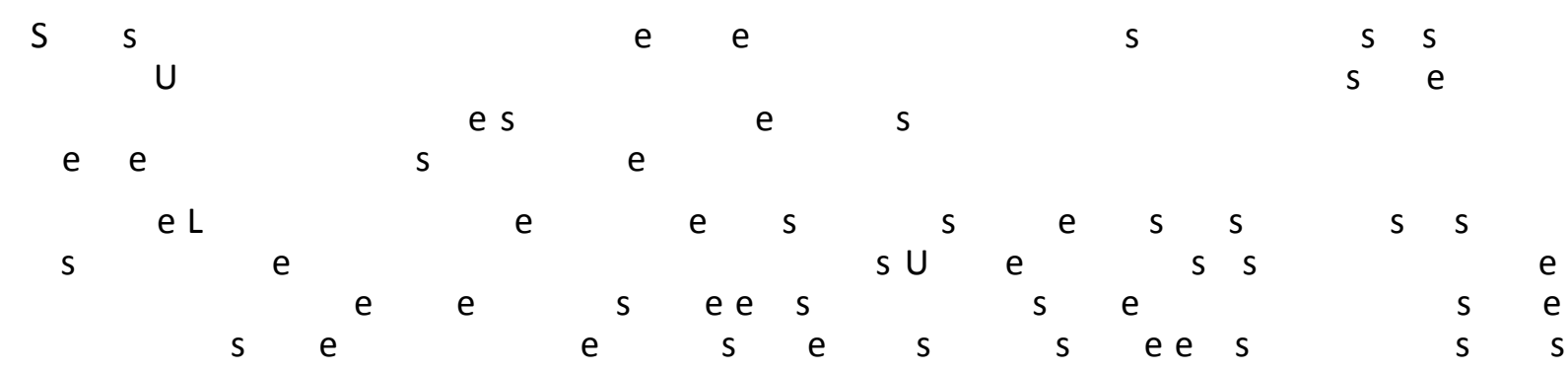


$\mathrm{E}$
Trends and Issues. 7

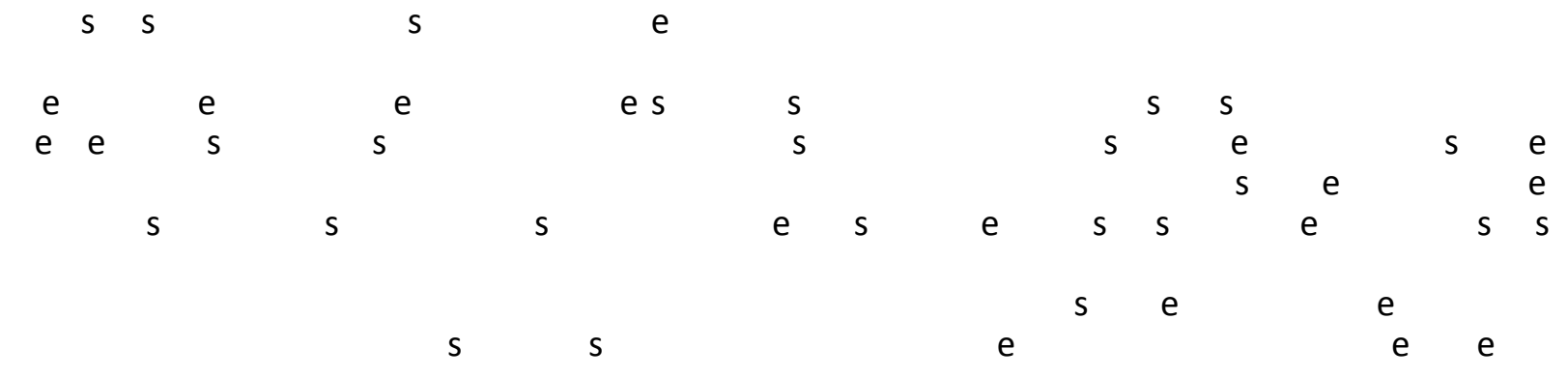

$\mathrm{S}$<smiles>[SiH3][SiH3]</smiles> 


$$
\begin{aligned}
& \text { S } \\
& \text { S } \\
& \text { S S } \\
& \text { s s e s } \\
& \text { e ese } e^{e} \\
& \text { e } \\
& \text { e e s } \\
& \text { e s } \\
& \text { e } \\
& \text { e } \\
& \text { e } \\
& e^{s} s \\
& \text { e } \quad s^{e} \text { e } \\
& \begin{array}{lllll} 
& s & & \text { se } \\
& S & S
\end{array} \\
& \text { e s } \\
& \mathrm{S} \\
& \text { e e } \\
& \text { e s s }
\end{aligned}
$$

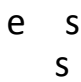

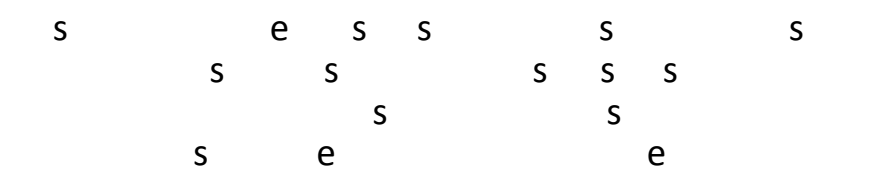

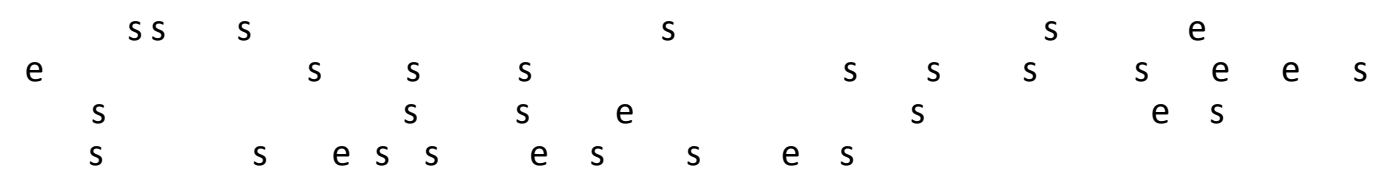

\section{Methodology}

\subsection{Participants}
S
S
S SD

\subsection{Instruments}

$U S S$ SS

e

s e

Eating behaviour patterns questionnaire (Schlundt, Hargreaves \& Buchowski, 2003): U

$$
\mathrm{s}
$$

S

$\begin{array}{llll}S & S & & \\ & & S & s\end{array}$
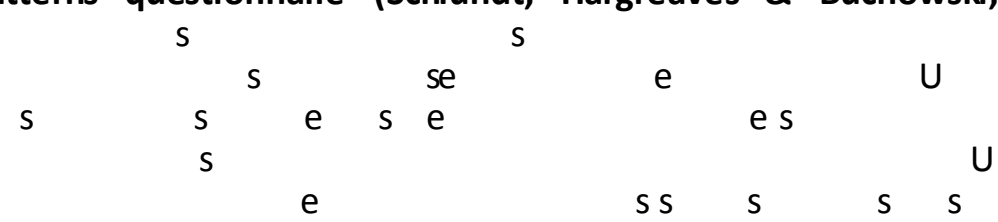

$\mathrm{S}$

Eating and appraisal due to emotions and stress questionnaire

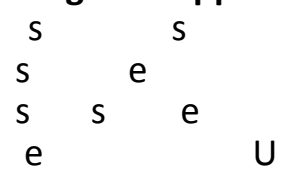

$\mathrm{U}$ : U $\mathrm{S}$ es $s$ S

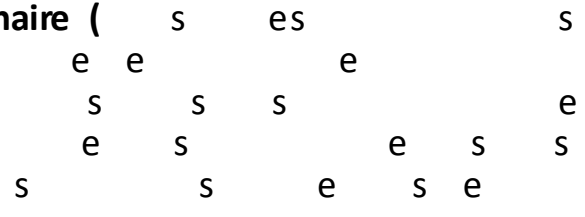




\section{$\mathrm{S}$}

S S

$S \quad S \quad S \quad S \quad S \quad S$

Big five inventory (John \& Srivastava, 1999): U

e $\mathrm{S} S$

$\mathrm{S}$

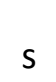

S

s e s e

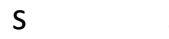

$$
\mathrm{S}
$$

S S

Paradise \& Kernis, 1999) U
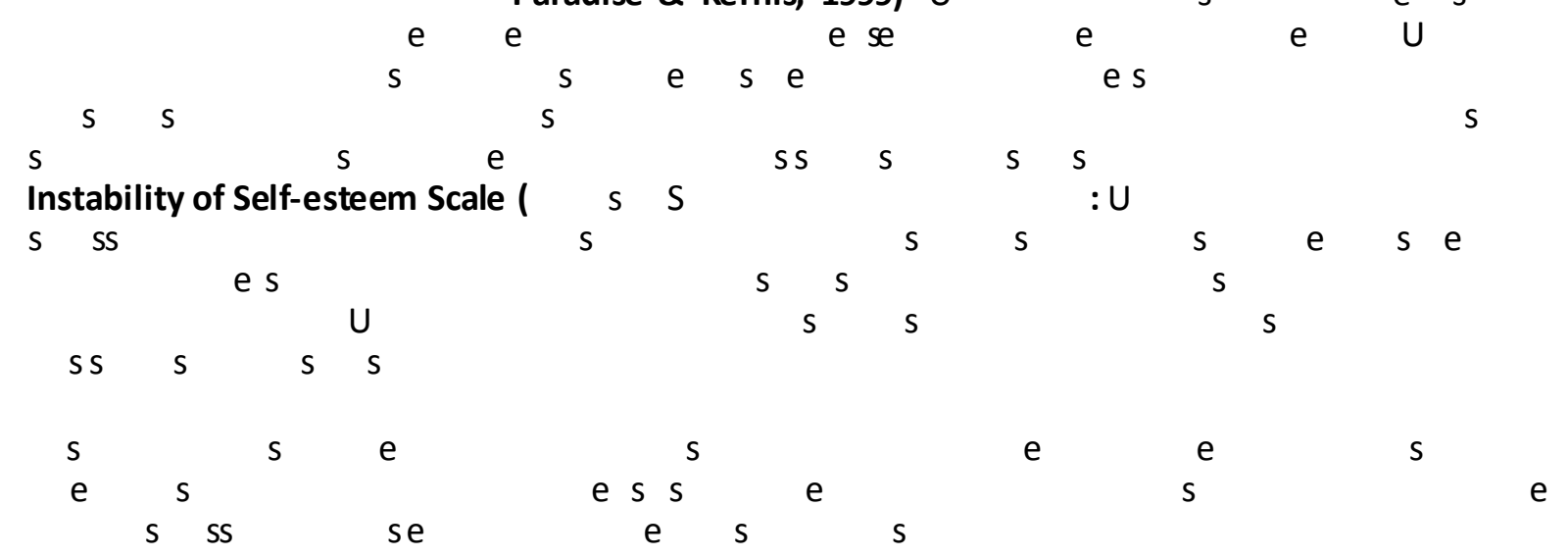

2.3. Procedure

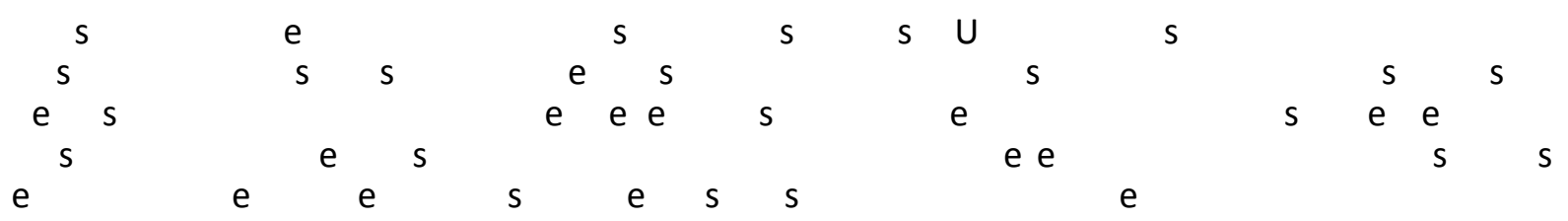

2.4. Statistics

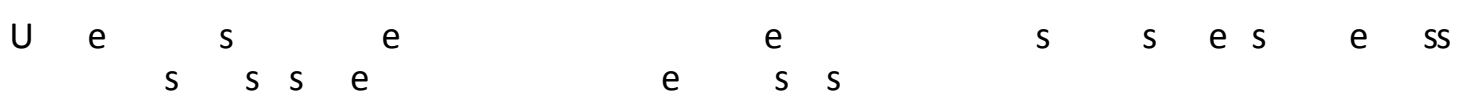

3. Results and Discussion

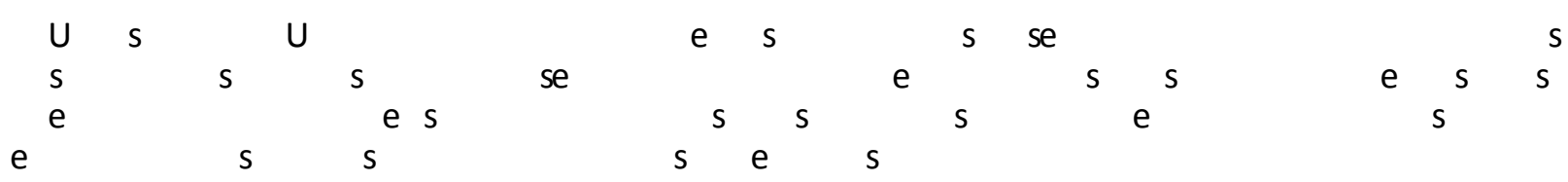


$\mathrm{E}$
Trends and Issues. 7

e

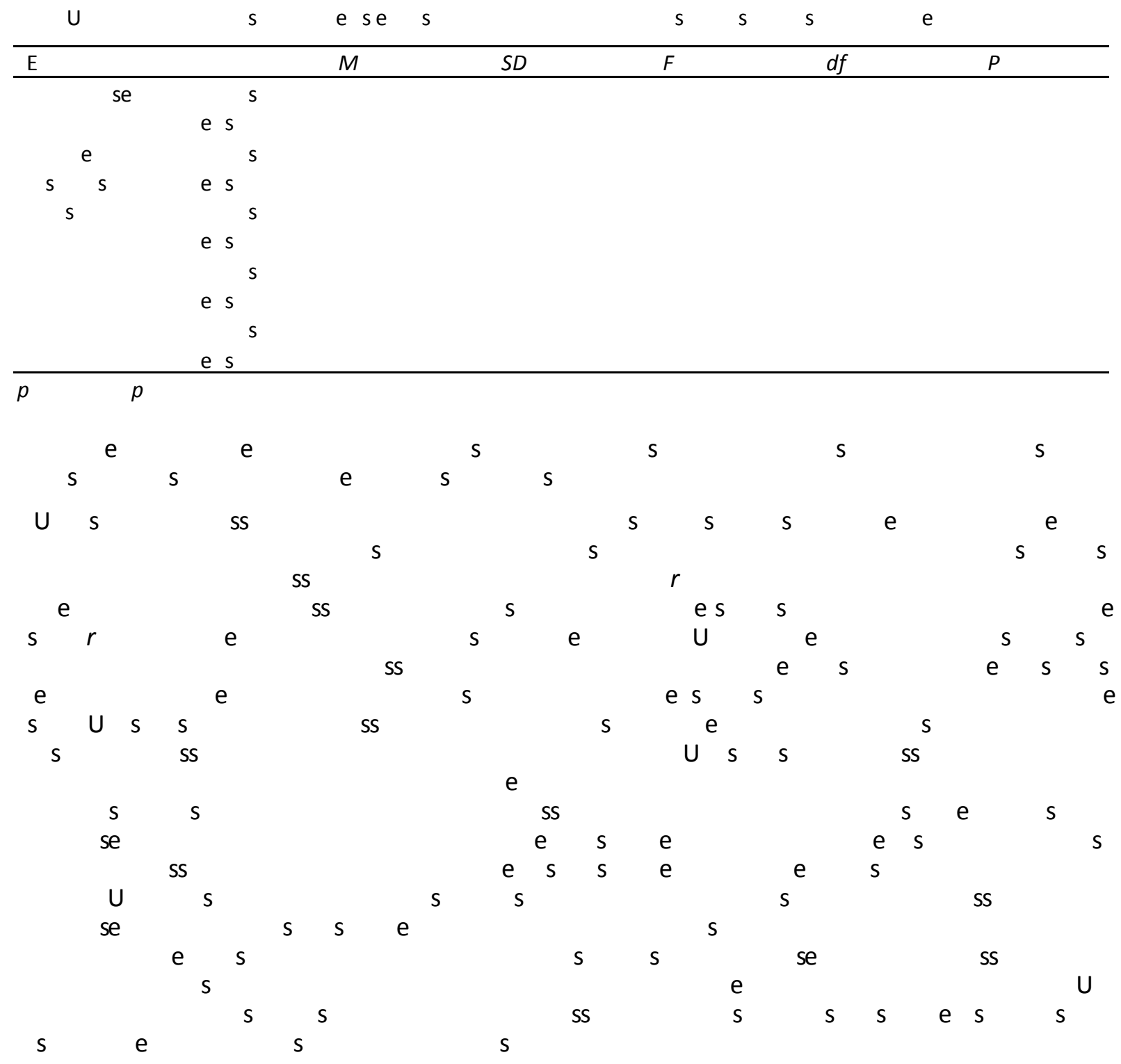

\section{Conclusion}

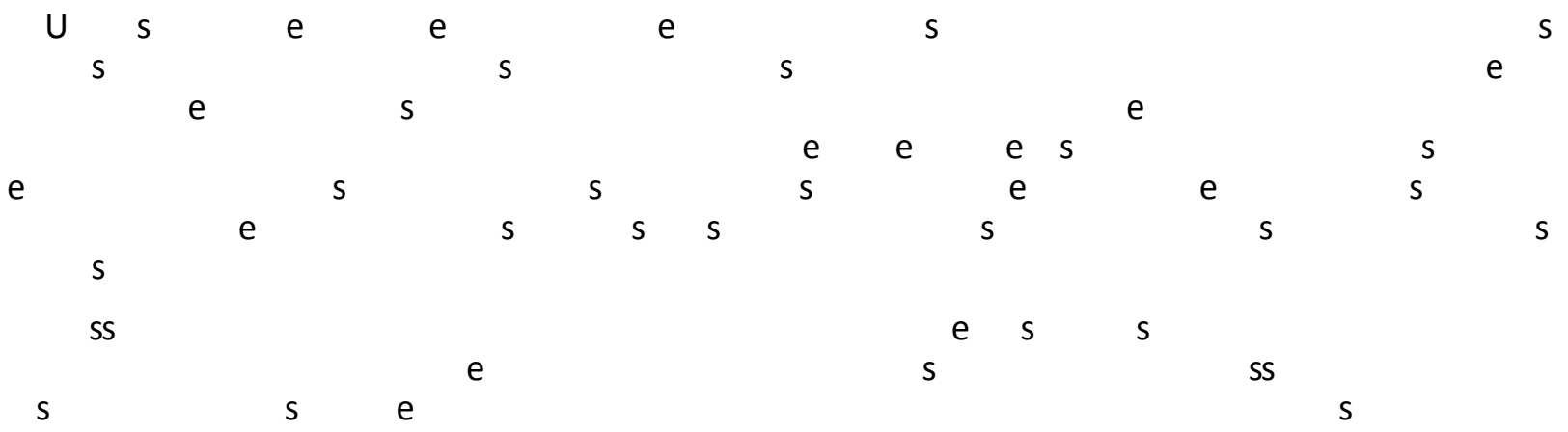


$\mathrm{S}$

e

S S

S S

S
S

$\mathrm{s}^{\mathrm{e}} \mathrm{s}$

$\mathrm{S}$ $\begin{array}{llll} & e & & e \\ s & s & s \\ & & & e\end{array}$

S
$\mathrm{S}$

$\mathrm{S}$ e s $s$

S S

$S$

\section{References}

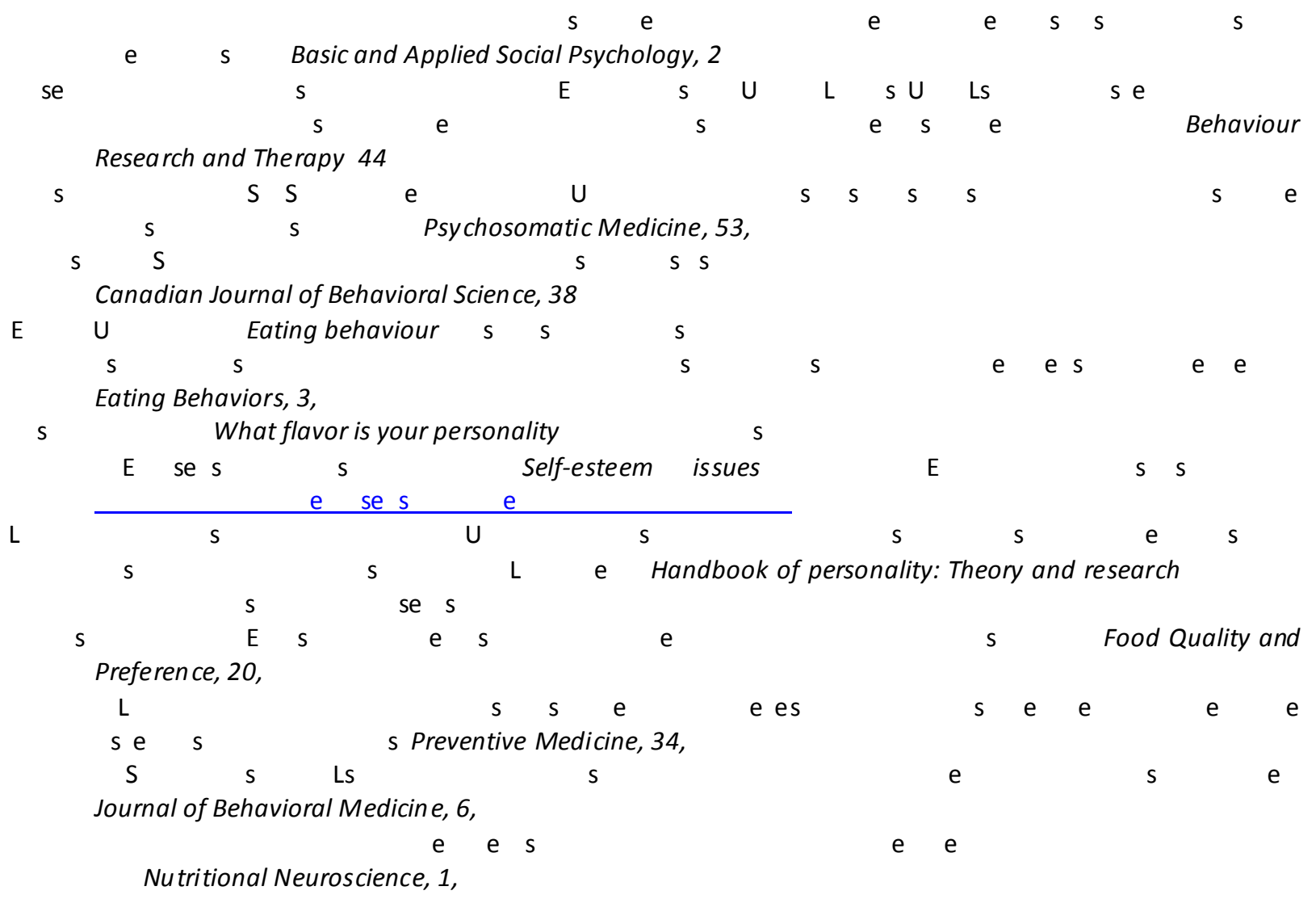

\section{e s e Appetite, 35,}

e L The psychology of eating: From healthy to disordered behavior se

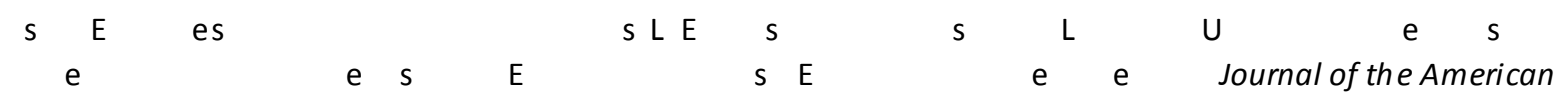
Dietetic Association, 107 s e

$\mathrm{s}$

$S$

e $E \quad S S$

$\mathrm{s}$ e e s

103
Development of the contingent self-esteem scale.

S

U $\quad$ s $\quad s \quad s$ Journal of the American Dietetic Association, 
$\begin{array}{ccccc}\mathrm{Ls} & \mathrm{L} & & \\ \mathrm{e} & \mathrm{s} & \mathrm{s} \\ \text { psychological health } & \mathrm{S} & \mathrm{s} & \mathrm{e}\end{array}$

E $S$

of Affective Disorders, 32,
U

e

e e e

s

Ls e Progress in the study of physical and

e

e

Journal 